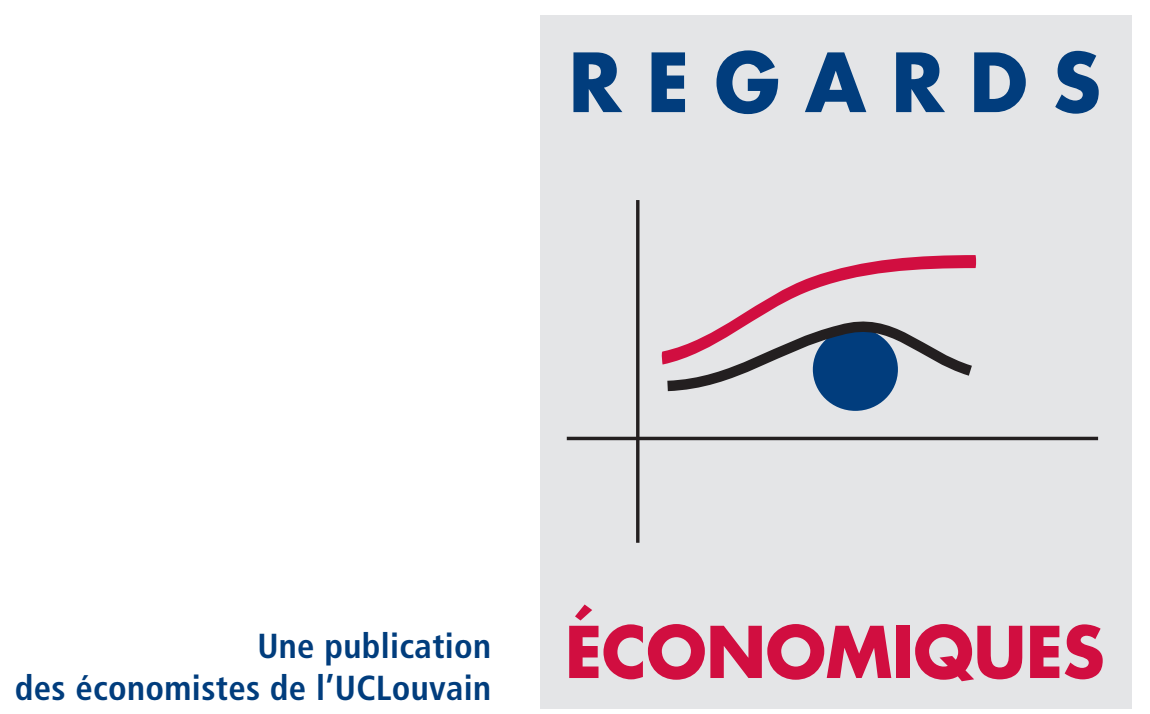

Juillet 2020 • Numéro 156

\title{
Perspectives économiques 2020-20211
}

L'éclatement de l'épidémie de coronavirus en début d'année a mis brutalement à l'arrêt l'activité économique d'un grand nombre de pays. Le choc économique est particulièrement sévère pour la Belgique, dont l'activité économique devrait enregistrer en première partie de cette année son recul le plus important depuis la seconde guerre mondiale.

\section{Service d'analyse économique de l'IRES}

A la faveur de la levée des mesures de confinement prises pour enrayer la propagation du virus, l'activité économique a commencé progressivement à reprendre dans de nombreux pays, en ce compris la Belgique et les autres pays européens. Les résultats des dernières enquêtes de conjoncture témoignent de cette reprise. Ainsi, après avoir très lourdement chuté en avril, la confiance des entreprises et des ménages belges a commencé à se redresser en mai, et elle a continué à se raffermir en juin.

La poursuite de la reprise économique en cours durant la seconde partie de l'année et en 2021 dépendra étroitement de l'évolution de la situation sanitaire. Il est ici fait l'hypothèse que, à l'avenir, la propagation du virus pourra être contrôlée sans que cela nécessite l'instauration d'une nouvelle période de confinement généralisé. Sous cette hypothèse, l'activité économique devrait rebondir fortement en seconde partie de cette année, avant de retrouver des rythmes trimestriels de croissance plus conventionnels en 2021.

Malgré la reprise attendue, l'activité économique en Belgique se situerait fin 2021 en dessous du niveau auquel elle se serait située sans la crise. Bien que courte, la récession actuelle devrait laisser des cicatrices profondes, notamment en termes d'emploi et de chômage, qui limiteront les capacités de redémarrage de l'économie.

Selon ce scénario, l'économie belge devrait se contracter de près de $10 \%$ en 2020. Elle afficherait à nouveau une croissance positive en 2021, de 7,5\% en base annuelle.

Malgré la mise en place de dispositifs de chômage temporaire, le choc économique provoqué par la crise épidémique devrait avoir un lourd impact sur la situation du marché du travail.

\footnotetext{
${ }^{1}$ Le contenu de cet article résume l'étude de conjoncture publiée par le Service d'analyse économique de l'IRES dans le numéro de juillet 2020 de Analyses économiques et prévisions. Des renseignements sur cette étude peuvent être obtenus sur https:/uclouvain.be/en/research-institutes/lidam/ires/service-d-analyse-economique.html
} 


\begin{tabular}{|c|c|c|}
\hline & $\begin{array}{l}\text { Résumé des } \\
\text { prévisions pour la } \\
\text { Belgique }\end{array}$ & $\begin{array}{l}\text { - L'économie belge devrait se contracter de } \mathbf{9 , 9} \% \text { en } 2020 \text {. Elle retrouverait une } \\
\text { croissance positive, de } \mathbf{7 , 5} \% \text {, en } 2021 \text {. } \\
\text { - L'emploi diminuerait, en termes nets, de } \mathbf{1 0 6 . 0 0 0} \text { unités en } 2020 \text {. En 2021, il } \\
\text { augmenterait de } \mathbf{3 2 . 0 0 0} \text { unités. } \\
\text { - Le nombre de demandeurs d'emploi augmenterait de } \mathbf{1 3 2 . 8 0 0} \text { unités en } 2020 \text {. } \\
\text { En } 2021 \text {, il diminuerait de } \mathbf{4 . 7 0 0} \text { unités. } \\
\text { - L'inflation générale des prix à la consommation s'élèverait à } \mathbf{0 , 9} \% \text { en } 2020 \text { et } \\
\mathbf{1 , 7} \% \text { en } 2021 \text {. } \\
\text { - Le déficit budgétaire des administrations publiques atteindrait } \mathbf{1 1 , 5} \% \text { du PIB en } \\
2020 \text { et } \mathbf{6 , 6} \% \text { en } 2021 \text {. }\end{array}$ \\
\hline
\end{tabular}

\section{Recul historique de l'activité économique mondiale}

L'éclatement au début de l'année de l'épidémie mondiale de coronavirus (COVID-19) a plongé soudainement l'économie mondiale dans une profonde récession, probablement la plus importante depuis la Grande Dépression des années 1930. Elle est la conséquence directe des mesures de confinement que de nombreux pays ont dû prendre dans le but d'endiguer la propagation du virus. En ce sens, selon les mots de Baldwin, la récession économique actuelle est «intentionnelle et inévitable» ${ }^{2}$.

L'arrêt de l'activité économique a débuté à des moments différents selon les régions. Ainsi, alors que l'activité économique fut bloquée dès janvier en Chine et dans plusieurs autres pays asiatiques, ce n'est qu'à partir de la mi-mars que l'Europe et le continent américain furent confrontés à une situation similaire. En Chine, la levée des mesures de confinement a débuté en mars. Celle-ci est à présent en cours dans de nombreux autres pays, dont notamment tous les pays de la zone euro et les Etats-Unis. L'activité économique mondiale commence dès lors à reprendre, mais son niveau reste nettement inférieur à ce qu'il était avant l'éclatement de la crise économique.

Le choc économique mondial subi en ce début d'année est considérable. L'activité économique de l'ensemble des pays de l'OCDE a ainsi reculé de 1,8\% au premier trimestre. Au sein de ce groupe, le recul du PIB atteint 3,6 \% pour les pays de la zone euro et $1,3 \%$ pour les Etats-Unis. Du côté des pays émergents, l'activité économique accuse une baisse de $9,8 \%$ en Chine au premier trimestre. A l'échelle mondiale, le recul du PIB est de 3,0\% au premier trimestre 3 .

Les données sur la production industrielle et le commerce international indiquent que le recul de l'activité économique mondiale s'est nettement amplifié au début du deuxième trimestre. Ainsi, en avril, la production industrielle mondiale était en baisse de $12 \%$ par rapport à son niveau un an plus tôt tandis que les importations mondiales de marchandises étaient de $16 \%$ inférieures à leur niveau un an plus tôt. Ces chiffres sont du même ordre de grandeur que ceux enregistrés au plus fort que la crise économique et financière de 2008-2009.

Alors que la croissance économique chinoise devrait redevenir positive au deuxième trimestre, celle des pays les plus avancés devrait en revanche être plus négative qu'au premier trimestre, la période de confinement ayant débuté en mars ou avril pour un grand nombre de ces pays. Ainsi, selon certains modèles de prévisions en temps réel, le PIB réel américain aurait reculé d'environ $10 \%$ entre le

\footnotetext{
${ }^{2}$ Source: Baldwin, Richard, “The supply side matters: Guns versus butter, COVID-style”, VOX CEPR Policy Portal, 22 March 2020.

${ }^{3}$ Les chiffres de croissance indiqués dans le paragraphe indiquent la variation, en pourcentage, d'un trimestre donné par rapport au trimestre précédent.
} 


\section{... Recul historique de l'activité économique mondiale}

premier et le deuxième trimestre. Pour la zone euro, le recul du PIB réel pourrait atteindre $14 \%$ au deuxième trimestre.

Au moment de clôturer cette étude, il apparaît qu'un grand nombre de pays ont procédé à la levée plus ou moins complète des mesures de confinement. Une reprise plus généralisée de l'activité économique au niveau mondial devrait donc avoir lieu durant la seconde partie de l'année. Il ne devrait toutefois s'agir que d'une reprise partielle, qui ne permettra pas de compenser intégralement les pertes d'activité enregistrées durant la première partie de l'année. D'une part, le maintien probable de mesures de distanciation physique nécessaires pour empêcher une nouvelle vague de contamination ne permettra pas à plusieurs secteurs, en particulier celui de l'horeca, celui des arts, spectacles et services récréatifs et celui du transport aérien, de retrouver une activité normale. D'autre part, l'arrêt brutal de l'activité économique durant près de 2 mois a fragilisé considérablement l'économie mondiale. Ainsi, en mai, l'indicateur mondial des directeurs d'achats portant sur l'activité de l'industrie et des services était à peine de 36,3 alors que le seuil critique qui marque la frontière entre l'expansion et la récession est de 50. Une de ses composantes les plus faibles est celle portant sur les nouvelles commandes à l'exportation, dont le niveau était de 31,7 en mai. La dégradation de la situation conjoncturelle demeure très marquée dans les pays les plus avancés, l'indicateur des directeurs d'achats pour ces pays étant de 33,2 en mai, contre 42,7 pour les pays émergents ( 54,5 pour la Chine). Outre la dégradation du climat des affaires, il convient également de noter que la confiance des ménages s'est effondrée dans un grand nombre de pays. Ainsi, en zone euro, l'inquiétude des ménages par rapport au risque de chômage a grimpé en mai à son niveau le plus élevé depuis la Grande Récession de 2008-2009. La situation est encore plus dramatique aux Etats-Unis où le taux de chômage $\mathrm{a}$ atteint un record historique de $13,3 \%$ en mai, alors qu'il était de seulement $3,5 \%$ en février.

Selon notre scénario d'une récupération incomplète de l'activité économique durant la seconde partie de l'année en cours, nous anticipons pour l'ensemble de 2020 une contraction de l'activité économique de l'ordre de $8 \%$ aux Etats-Unis et de près de $9 \%$ en zone euro (tableau 2). Dans ses dernières prévisions, l'OCDE annonce par ailleurs une croissance économique de - 6,0\% au niveau mondial et de - 7,5\% pour le groupe des pays $\mathrm{OCDE}^{4}$. La croissance économique de la Chine serait positive, mais d'à peine $1,0 \%$. Une contraction historique du commerce international est également attendue qui, selon nos prévisions, atteindrait près de - 10,0 \% (tableau 2).

Les perspectives économiques pour 2021 sont fortement incertaines. D'une part, l'évolution de la situation économique demeure étroitement dépendante de l'évolution de la crise épidémique. D’autre part, elle dépendra également des séquelles laissées par la récession actuelle en termes de pertes d'emplois et de pouvoir d'achat, de hausse du chômage et de faillites d'entreprises, d'augmentation de l'endettement et de durcissement des conditions financières.

Notre scénario de base pour 2021 repose sur l'hypothèse que, pour ce qui concerne la crise épidémique, une deuxième vague de contamination pourra être évitée sans que cela nécessite une nouvelle période de confinement généralisé. Des mesures de distanciation physique resteront toutefois d'application, qui auront pour effet d'empêcher le retour à une activité normale dans plusieurs secteurs d'activité économique. Sur le plan économique, il est supposé que les dégâts économiques provoqués par la récession actuelle auront pour effet de limiter l'expansion économique mondiale l'an prochain. Les nombreuses mesures prises au plan monétaire et budgétaire pour relancer l'activité économique devraient toutefois progressi-

\footnotetext{
${ }^{4}$ Source: Perspectives économiques OCDE, Volume 2020 Issue 1, No107, OCDE.
} 


\section{... Recul historique de l'activité économique mondiale}

Tableau 1.

Hypothèses (données fin de période)

Sources : Macrobond, Belgostat Prévisions : IRES

\footnotetext{
* Hypothèse

'Baril de Brent

${ }^{2}$ Taux d'intérêt sur les fonds fédéraux

${ }^{3}$ Taux repo de la BCE
}

\section{Forte récession de l'économie belge}

vement ramener l'économie mondiale sur une trajectoire de croissance au moins équivalente à celle qui prévalait avant la crise. Malgré tout, le niveau d'activité économique en 2021 resterait inférieur à ce qu'il aurait été en l'absence de crise.

Selon ce scénario de base, l'expansion économique attendue en 2021 ne permettra donc pas de combler le creux économique de cette année. La croissance économique attendue serait ainsi, en base annuelle, d'environ 2,0 \% aux Etats-Unis, $6,0 \%$ en zone euro et $8,0 \%$ en Chine (tableau 2). Dans ce contexte, la croissance du commerce international rebondirait à 5,5\% (tableau 2). L'OCDE, sur la base d'un scénario similaire, prévoit pour sa part un rebond de la croissance économique mondiale à 5,2\% en 2021 .

Après être descendu à 19 \$ à la fin avril, le prix du baril de Brent a ensuite connu une lente remontée, qui l'a ramené aux alentours de 40 \$ en juin. Cette remontée est due pour partie au redémarrage de l'activité économique dans plusieurs régions, en particulier en Chine qui est un gros consommateur de produits pétroliers. Elle s'explique ensuite par l'accord conclu par la Russie et les pays membres de l'OPEP, portant sur une réduction de leur production jointe de 9,7 millions de barils par jour au moins jusqu'à la fin de l'année. Selon notre scénario d'une poursuite de la reprise économique mondiale, la demande mondiale de pétrole devrait continuer à se raffermir sur la période de projection. Malgré cela, et en dépit des mesures de limitation de la production prises par certains grands producteurs, l'Association internationale de l'énergie estime que l'offre de pétrole restera excédentaire sur la période de projection. Dans ce contexte, il est fait l'hypothèse que le prix du baril de Brent s'établira à un niveau moyen de 41 \$ en 2020 et 44 \$ en 2021 (tableau 1).

\begin{tabular}{lcccccc}
\hline & 2016 & 2017 & 2018 & 2019 & $2020^{\star}$ & $2021^{*}$ \\
\hline Prix du pétrole (\$ par baril) & 43,7 & 54,2 & 71,1 & 66,0 & 41,0 & 45,0 \\
\hline $\begin{array}{l}\text { Taux de change } \\
\text { Euro/US \$ (fin de période) }\end{array}$ & 1,05 & 1,19 & 1,14 & 1,11 & 1,12 & 1,12 \\
\hline $\begin{array}{l}\text { Toux de politique monétaire } \\
\text { (fin de période) } \\
\quad \text { Etats-Unis }\end{array}$ & 0,75 & 1,50 & 2,50 & 1,75 & 0,00 & 0,00 \\
$\quad$ Zone euro $^{3}$ & 0,00 & 0,00 & 0,00 & 0,00 & 0,00 & 0,00 \\
\hline
\end{tabular}

Comme dans de nombreux autres pays, le déclenchement de la pandémie de COVID-19 a contraint les autorités belges à confiner sa population durant plusieurs semaines pour tenter d'enrayer la propagation du virus. La phase de confinement a débuté le 18 mars et a commencé à être relâchée à partir du 4 mai. Durant cette phase, des pans importants de l'économie se sont retrouvés à l'arrêt ou ont fonctionné au ralenti, plongeant l'économie dans sa plus grave récession depuis la seconde guerre mondiale. Ainsi, selon les données les plus récentes des comptes nationaux, l'économie belge s'est contractée de 3,6\% au $1^{\text {er }}$ trimestre (par rapport au trimestre précédent), et nos estimations pour le deuxième trimestre indiquent une contraction historique de près de $20 \%$ pour ce trimestre (par rapport au trimestre précédent). Le chômage temporaire a explosé durant cette période, cette situation concernant près d'un million de salariés à la fin avril, soit environ $25 \%$ de la population active salariée. 


\section{... Forte récession de l'économie belge}

Depuis le début mai, les mesures de confinement ont été progressivement levées, permettant une reprise partielle de l'activité économique. La poursuite de la reprise durant le reste de l'année en cours et en 2021 dépendra de manière critique de l'évolution de la situation sanitaire en Belgique et à l'étranger. Il est ici fait l'hypothèse que la propagation du virus continuera à être maîtrisée et que le redressement de la situation économique ne sera pas bloqué par l'imposition de nouvelles mesures de confinement en raison d'un rebond de l'épidémie.

Sous cette hypothèse, une reprise vigoureuse de l'activité économique devrait avoir lieu durant la seconde partie de cette année (graphique 1). Cette reprise ne devrait toutefois que compenser partiellement les pertes d'activité subies durant la première partie de l'année. D'une part, le contrôle de l'épidémie impose le maintien de règles sanitaires, la plus contraignante étant probablement le respect d'une distanciation sociale, qui empêcheront le retour d'une activité normale dans plusieurs secteurs d'activité, en particulier le secteur de l'horeca, celui du transport aérien et celui de la culture et des loisirs. D'autre part, l'économie belge et celle de ses principaux partenaires commerciaux sortent de la phase de confinement en étant fortement fragilisées. Ainsi, en Belgique, la confiance des ménages et celle des entreprises se sont effondrées au début du deuxième trimestre, et malgré une évolution plus positive en mai et en juin, elles se trouvaient toujours à un niveau historiquement bas à la fin du deuxième trimestre (graphique 2). Du côté des ménages, on constate en particulier une hausse dramatique de l'inquiétude par rapport au risque de chômage tandis que les entreprises, quel que soit leur secteur d'activité, font le constat d'une activité fortement déprimée et se montrent très pessimistes par rapport à l'évolution future de leur activité. C'est ainsi que, fin juin, selon les résultats des enquêtes menées hebdomadairement par l'Economic Risk Management Group (ERMG) de la BNB depuis le début de la crise, $5 \%$ des entreprises interrogées indiquaient un risque important de faillites au cours des prochains mois. Par ailleurs, malgré la reprise de l'activité économique, de nombreuses entreprises avaient encore une proportion importante de leurs travailleurs en chômage temporaire.

$\mathrm{Si}$, comme nous le supposons, une résurgence de l'épidémie est évitée, la reprise économique devrait se consolider en 2021. L'allure de la reprise redeviendrait plus modérée (graphique 1), l'économie belge convergeant progressivement vers sa trajectoire de croissance d'avant crise.
Graphique 1. Trajectoire du PIB réel de la Belgique : 2019-2021

Source : ICN Calculs personnels

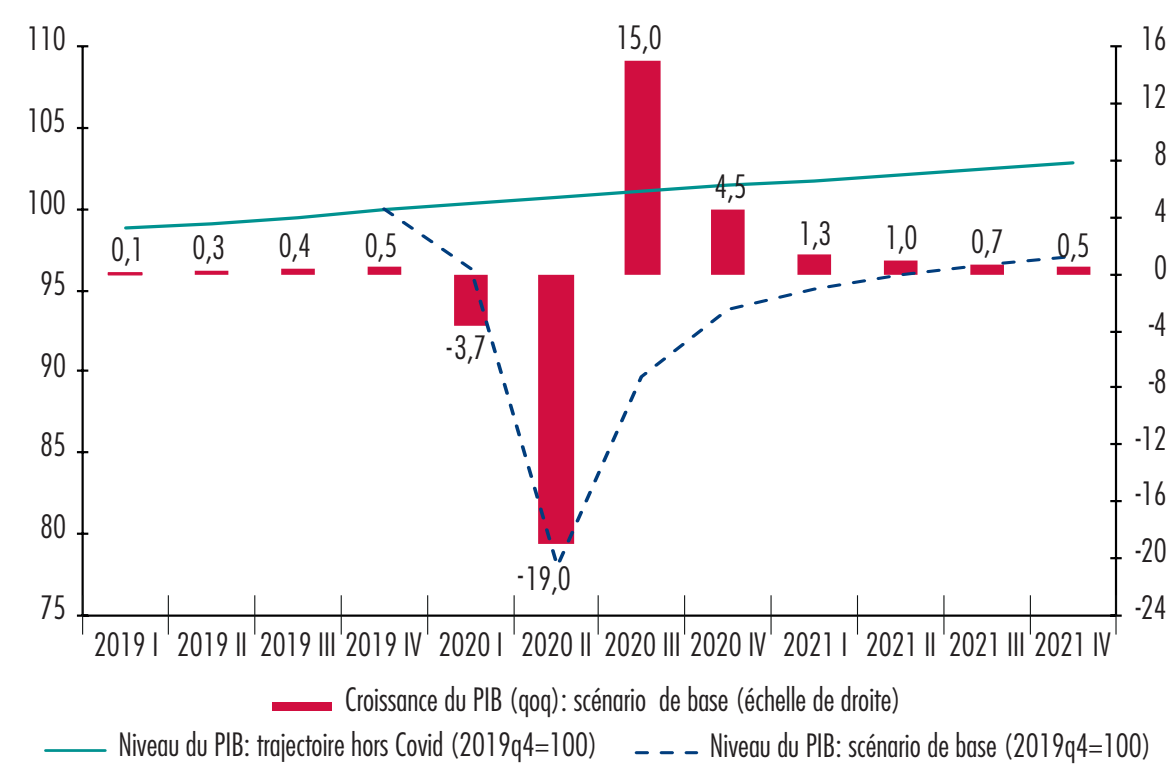


Graphique 2. Confiance des ménages et des entreprises (en moyenne centrée réduite)

Source : $B N B$ Calculs : IRES

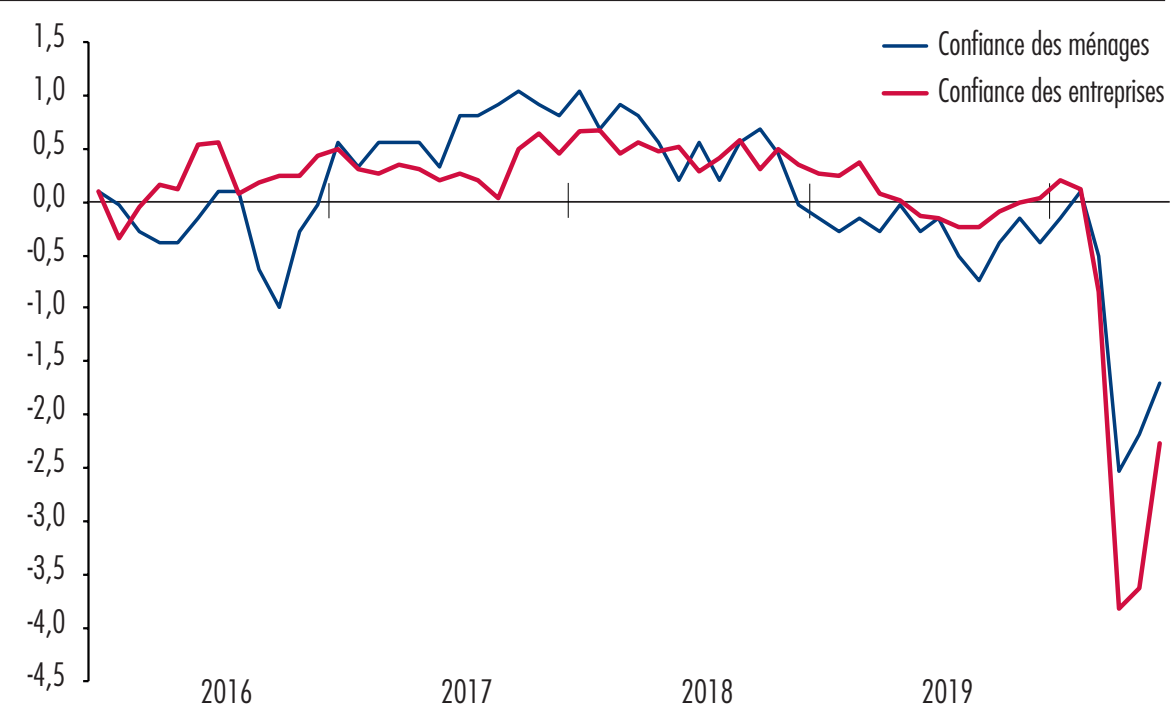

Selon ces perspectives, l'activité économique belge accuserait, en base annuelle, une contraction de près de $10,0 \%$ en 2020 (tableau 2). Elle retrouverait une croissance positive en 2021, de 7,5\%. Fin 2021, le niveau d'activité économique serait donc inférieur à ce qu'il aurait été sans l'éclatement de la crise.

La consommation privée s'est contractée de 6,5\% au $1^{\text {er }}$ trimestre 2020 (par rapport au trimestre précédent) et, en raison du confinement, elle accusera un recul encore plus marqué au $2^{\mathrm{e}}$ trimestre. Une reprise vigoureuse des dépenses des ménages est en revanche attendue au $3^{\mathrm{e}}$ trimestre, celle-ci étant en partie soutenue par un rattrapage des dépenses n'ayant pu être réalisées durant la période de confinement. Cette phase de rattrapage ne sera que temporaire, si bien que la croissance des dépenses de consommation devrait par la suite se normaliser. La reprise attendue devrait toutefois être partielle et graduelle, de sorte que la consommation ne devrait pas retrouver sur la période de projection le niveau qu'elle aurait atteint sans la crise. La confiance des ménages a en effet été fortement ébranlée par la crise, la principale source d'inquiétude des ménages étant liée à la dégradation de la situation du marché du travail. Le chômage temporaire a en effet fortement augmenté depuis mars et de nombreux licenciements sont à craindre au cours des prochains mois. Par ailleurs, malgré les mesures prises par les pouvoirs publics pour atténuer les pertes d'emploi et éviter une trop forte dégradation de la situation financière des ménages, de nombreux particuliers ont subi des pertes de revenus. Ainsi, au niveau macroéconomique, le revenu disponible réel des particuliers devrait se contracter de 2,3\% en 2020. Il augmenterait en revanche de $2,7 \%$ en 2021. Dans ce contexte, la consommation des particuliers diminuerait, en base annuelle, de 10,6\% en 2020 et sa récupération en 2021 serait de $8,9 \%$.

Pour ce qui concerne l'investissement des entreprises, celui-ci a reculé de 3,6 \% au $1^{\text {er }}$ trimestre 2020 (par rapport au trimestre précédent). Une baisse nettement plus sévère devrait avoir lieu au $2^{\mathrm{e}}$ trimestre, laquelle serait suivie d'un rebond en seconde partie d'année. Le rebond devrait cependant être moins important que celui attendu pour le PIB et les autres postes de la demande. Les déterminants économiques et financiers de l'investissement ne sont en effet pas très encourageants. Malgré le redémarrage de l'activité économique, il apparaît dans les dernières enquêtes de conjoncture que les entreprises continuent à être très pessimistes sur les perspectives économiques pour les prochains mois. Ces mêmes enquêtes montrent également que le taux d'utilisation des capacités de production industrielle a fortement diminué durant la première partie de l'année et, compte tenu d'un redressement de l'activité économique qui devrait être partiel et gra- 


\section{... Forte récession de l'économie belge}

duel, les capacités de production industrielle devraient rester fortement sous-utilisées au cours des prochains mois. L'arrêt brutal et prolongé de l'activité économique a par ailleurs fortement endommagé la situation financière des entreprises. Les liquidités de nombreuses entreprises ont ainsi fortement diminué. Quant aux possibilités de financement externe, celles-ci sont plus limitées qu'avant la crise en raison d'un fort resserrement des conditions d'accès au crédit bancaire. Dans ces conditions compliquées, l'investissement des entreprises chuterait, en base annuelle, de près de $17 \%$ en 2020 et il ne rebondirait que de $9,1 \%$ en 2021 .

Après avoir subi une forte contraction en première partie d'année, les exportations belges de biens et services devraient en revanche profiter à partir du $3^{e}$ trimestre de la reprise de l'activité économique en zone euro. Dans la mesure où la croissance économique en zone euro devrait suivre un schéma de reprise assez similaire à celui attendu en Belgique, la demande en provenance des principaux marchés d'exportation ne devrait cependant se rétablir que partiellement sur la période de projection et elle resterait dès lors inférieure à ce qu'elle aurait été sans la crise. Ainsi, alors qu'elles devraient chuter d'environ 12,0\% en 2020, les exportations belges de biens et services seraient en hausse de $10,9 \%$ en 2021. Compte tenu de cette évolution des exportations et de celle des postes de la demande intérieure privée présentée auparavant, les importations belges de biens et services seraient en recul de $11,5 \%$ en 2020 et en hausse de $10,4 \%$ en 2021. Il en découle que la contribution du commerce extérieur à la croissance serait négative en 2020 (- 0,4 point de pourcentage) mais qu'elle redeviendrait positive en $2021(+0,4$ point de pourcentage).

Après avoir augmenté durant 25 trimestres consécutifs, l'emploi a diminué, en termes nets, de 7.700 unités au $1^{\text {er }}$ trimestre de cette année. En raison du choc brutal sur l'activité économique, l'emploi devrait continuer à baisser au $2^{\mathrm{e}}$ trimestre, même si les mesures prises par le gouvernement en matière de chômage temporaire et de soutien financier aux indépendants (droit passerelle, primes) devraient permettre de limiter les destructions d'emplois. Malgré la reprise économique attendue, des pertes d'emplois importantes devraient encore avoir lieu durant la seconde partie de l'année, en raison d'une augmentation des faillites et d'un redressement seulement partiel de l'activité économique dans de nombreux secteurs. Il convient par ailleurs de souligner que l'incertitude très forte sur les perspectives économiques suscitent la frilosité des entreprises, dont les intentions d'embauches demeuraient faibles à la fin juin. Selon ces considérations, l'emploi diminuerait de 106.000 unités endéans 2020. ${ }^{5}$ Notre scénario de base, qui prévoit une consolidation de la reprise économique en 2021, nous amène également à prévoir une reprise de l'emploi l'an prochain. Celle-ci sera graduelle, de sorte que l'emploi augmenterait de seulement 32.000 unités endéans 2021.

Selon les statistiques les plus récentes portant sur le nombre de demandeurs d'emploi inoccupés, le chômage s'est relevé au cours des derniers mois, cette évolution mettant un terme à une longue période de baisse. Notre projection d'emploi implique que le chômage continuera à se relever sur la période de projection. Ainsi, sous l'hypothèse d'une croissance de la population active de $0,5 \%$ en 2020 et 2021 , le nombre de demandeurs d'emplois inoccupés augmenterait d'environ 133.000 unités endéans 2020, avant de reculer de 4.700 unités endéans $2021 .{ }^{6}$ Le taux de chômage harmonisé Eurostat passerait alors de 5,4 \% fin 2019 à 9,1 \% fin 2021.

Sous l'effet principalement de la forte baisse des prix du pétrole, la croissance des prix a ralenti durant la première partie de l'année. Le niveau de l'inflation

\footnotetext{
${ }^{5}$ Les chiffres mentionnés font référence à l'évolution de l'emploi endéans l'année, c'est-à-dire entre le début et la fin de l'année.

${ }^{6}$ Les chiffres mentionnés font référence à l'évolution du nombre de chômeurs endéans l'année, c'est-à-dire entre le début et la fin de l'année.
} 


\section{N La prévision... \\ щu au-delà du chiffre de \& croissance du PIB \\ 岌}

Source : IRES, Service d'analyse économique

Source : IRES, Service d'analyse économique

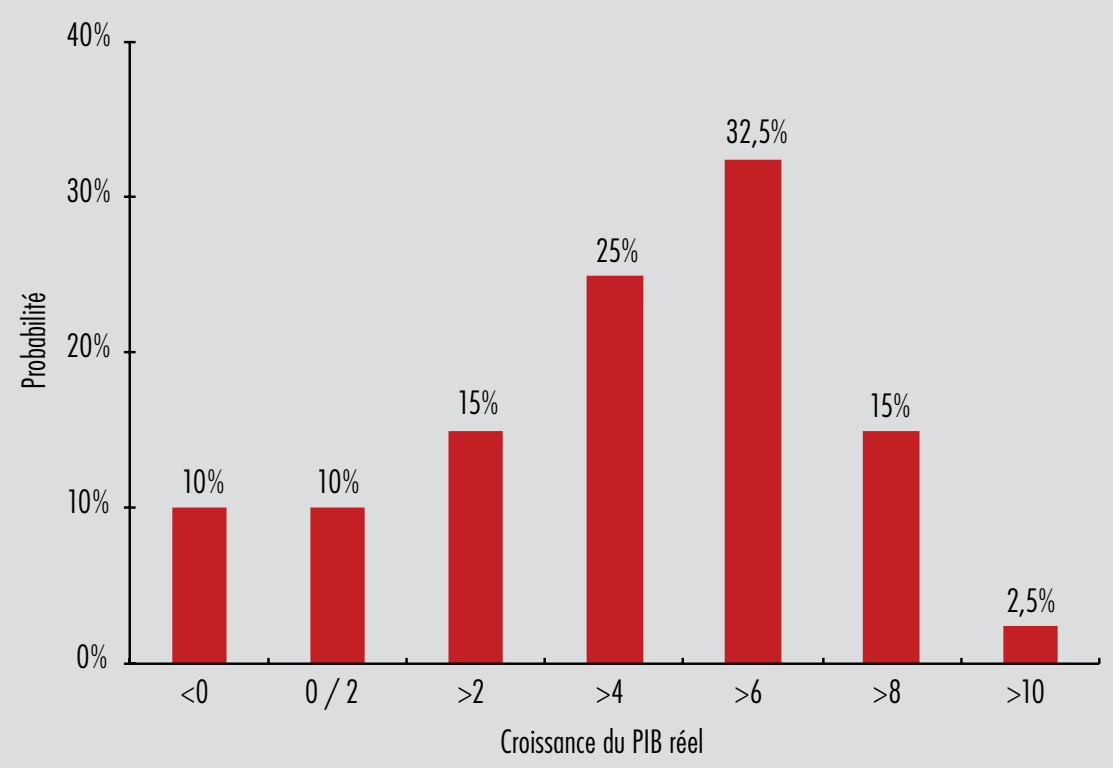
croissance du PIB est sans aucun doute l'élément central autour duquel s'articule notre projection. Même s'il est particulièrement commode de pouvoir résumer nos perspectives économiques en un seul chiffre, une telle pratique a néanmoins pour défaut de ne pas rendre compte de manière chiffrée de l'incertitude qui entoure n'importe quelle prévision. En présentant notre prévision sous la forme de probabilités attribuées à différents intervalles de croissance du PIB, les graphiques ci-dessous visent à combler cette lacune d'une manière simple*.
Comme c'est le cas pour beaucoup d'études conjoncturelles, le chiffre de

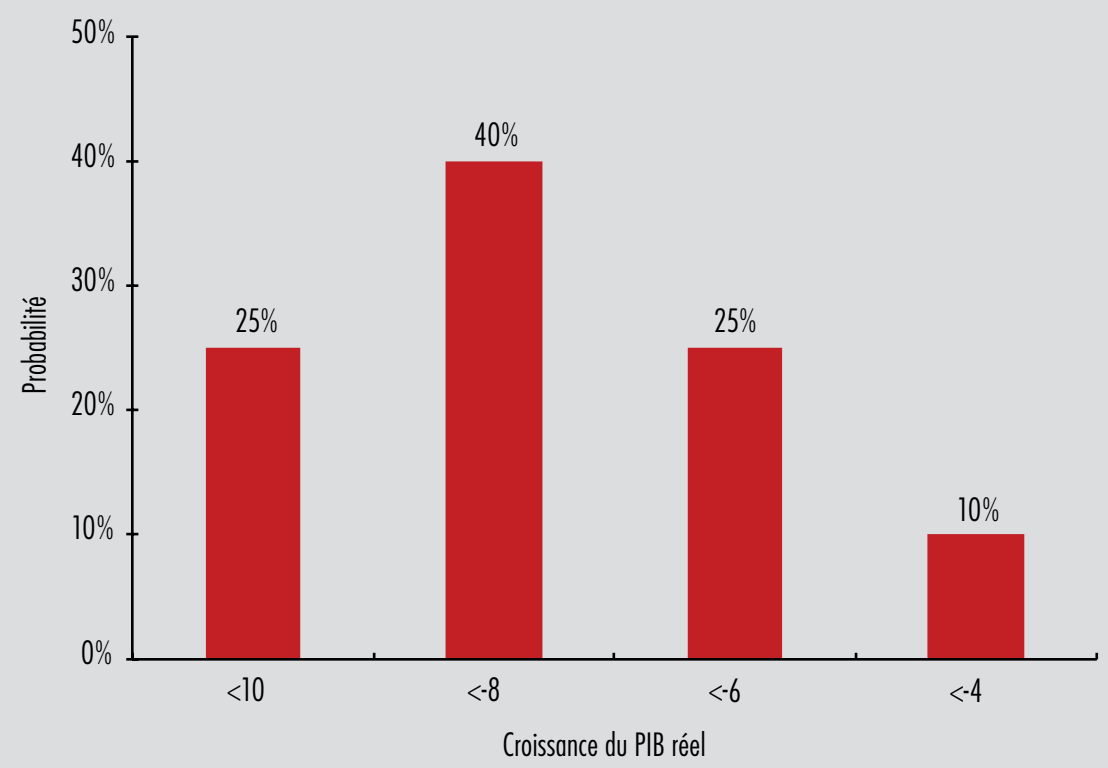

professionnels. 


\section{... Forte récession de l'économie belge}

générale a ainsi diminué à $0,5 \%$ en mai, avant de se relever à $0,6 \%$ en juin. Dans la foulée de la remontée des prix du pétrole, le niveau de l'inflation générale devrait également se relever au cours des prochains mois. Nous prévoyons ainsi, en base annuelle, une croissance moyenne de l'indice général des prix à la consommation de $0,9 \%$ en 2020 et de $1,7 \%$ en 2021 . Pour ce qui concerne l'indice santé, sa croissance annuelle moyenne serait de $1,1 \%$ en 2020 et $1,0 \%$ en 2021. L'indice-pivot, qui a été dépassé en février 2020, le serait à nouveau en mai 2021.
Tableau 2.

Prévisions de l'économie belge et internationale (pourcentage de variation par rapport à l'année précédente, sauf indications contraires)

Sources : ICN, Belgostat, INS Calculs et prévisions : IRES
' Contribution à la croissance du PIB

${ }^{2}$ Contribution à la croissance du PIB

${ }^{3}$ En pourcentage du revenu disponible

${ }^{4}$ Nombre total de chômeurs, en pourcentage de la population active; concept Eurostat

\begin{tabular}{|c|c|c|c|c|c|c|}
\hline Environnement international & 2016 & 2017 & 2018 & 2019 & $2020 p$ & $2021 \mathrm{P}$ \\
\hline PIB Etats-Unis & 1,6 & 2,4 & 2,9 & 2,3 & $-7,7$ & 2,2 \\
\hline PIB Zone euro & 1,9 & 2,7 & 1,9 & 1,2 & $-8,9$ & 5,9 \\
\hline PIB Chine' & 6,7 & 6,8 & 6,6 & 6,1 & 1,0 & 8,2 \\
\hline Commerce international & 2,3 & 5,6 & 3,3 & 1,4 & $-9,8$ & 5,5 \\
\hline
\end{tabular}

\begin{tabular}{|c|c|c|c|c|c|c|}
\hline Economie belge & 2016 & 2017 & 2018 & 2019 & 2020P & $2021 p$ \\
\hline PIB & 1,5 & 1,9 & 1,5 & 1,4 & $-9,9$ & 7,5 \\
\hline Consommation privée & 1,9 & 1,8 & 1,5 & 1,1 & $-10,6$ & 8,9 \\
\hline Consommation publique & 0,5 & 0,0 & 1,0 & 1,8 & 3,7 & 1,1 \\
\hline Investissement & 3,8 & 1,3 & 4,0 & 3,2 & $-15,2$ & 9,7 \\
\hline Entreprises & 5,0 & 1,6 & 3,9 & 3,2 & $-16,8$ & 9,1 \\
\hline Ménages & 2,4 & 0,1 & 1,0 & 4,9 & $-13,4$ & 11,7 \\
\hline Administrations & $-0,8$ & 1,0 & 10,9 & $-0,4$ & $-8,0$ & 9,9 \\
\hline $\begin{array}{l}\text { Demande intérieure } \\
\text { (hors variation des stocks) }\end{array}$ & 2,0 & 1,3 & 2,0 & 1,8 & $-8,4$ & 7,0 \\
\hline Variations des stocks ${ }^{2}$ & 0,2 & $-0,1$ & 0,3 & $-0,4$ & $-1,2$ & 0,0 \\
\hline Demande intérieure totale & 2,2 & 1,2 & 2,2 & 1,4 & $-9,5$ & 7,1 \\
\hline Exportations de biens et services & 6,5 & 5,3 & 1,2 & 1,1 & $-11,9$ & 10,9 \\
\hline Importations de biens et services & 7,5 & 4,4 & 2,1 & 1,0 & $-11,5$ & 10,4 \\
\hline Exportations nettes ${ }^{2}$ & $-0,7$ & 0,7 & $-0,7$ & 0,1 & $-0,4$ & 0,4 \\
\hline Prix à la consommation & 2,0 & 2,1 & 2,1 & 1,4 & 0,9 & 1,7 \\
\hline Indice "santé" & 2,1 & 1,8 & 1,8 & 1,5 & 1,1 & 1,0 \\
\hline Revenu disponible réel des ménages & 1,8 & 1,8 & 1,0 & 2,4 & $-2,3$ & 2,7 \\
\hline Taux d'épargne des ménages ${ }^{3}$ & 12,5 & 12,3 & 12,1 & 13,0 & 20,1 & 15,7 \\
\hline Emploi intérieur & 1,3 & 1,6 & 1,4 & 1,6 & $-0,6$ & $-0,7$ \\
\hline Demandeurs d'emploi inoccupés & $-4,4$ & $-5,0$ & $-5,8$ & $-3,8$ & 12,5 & 11,6 \\
\hline Taux de chômage ${ }^{4}$ & 7,9 & 7,1 & 6,0 & 5,4 & 7,2 & 9,3 \\
\hline $\begin{array}{l}\text { Solde net de financement des } \\
\text { administrations publiques } \\
\text { (en \% du PIB) }\end{array}$ & $-2,4$ & $-0,7$ & $-0,8$ & $-1,9$ & $-11,5$ & $-6,6$ \\
\hline
\end{tabular}




\section{Risques}

Plusieurs risques importants pourraient perturber de manière plus ou moins sévère la reprise de l'activité économique prévue pour la seconde partie de l'année et pour l'an prochain (Encadré 2). Le principal risque a trait à l'évolution de la situation épidémique et concerne la survenance possible à l'automne prochain d'une deuxième vague de contamination qui imposerait le rétablissement, même partiel, de mesures de confinement. Ensuite, les difficultés financières que pourraient connaître les entreprises et les ménages si la crise épidémique se prolonge pourraient mettre sous pression le système bancaire, tout particulièrement dans les pays où l'endettement des entreprises était déjà élevé avant la crise. Le système financier international peut également être fragilisé par une nouvelle crise de la dette des pays émergents qui, en plus d'être confrontés à la crise épidémique, souffrent d'un arrêt brutal des financements internationaux. Les conséquences économiques de la crise épidémique pourraient également induire un regain de tensions commerciales entre la Chine et les Etats-Unis, de même qu'elles pourraient compliquer les négociations commerciales post-Brexit entre l'Europe et le Royaume-Uni.

Cette étude a été réalisée sous la direction de Vincent Bodart, avec la collaboration de Gautier Attanasi, François Courtois, Sébastien Fontenay, Nathan Lachapelle, Alexandre Ounnas et Mathieu Sauvenier. A l'exception de Gautier Attanasi et Mathieu Sauvenier, tous les auteurs sont chercheurs à l'IRES. Gautier Attanasi et Mathieu Sauvenier sont chercheurs au CORE.

REGARDS ÉCONOMIQUES

Place Montesquieu, 3 B1348 Louvain-la-Neuve regard-ires@uclouvain.be tél. 010473426 www.regards-economiques.be
Directeur de la publication: Vincent Bodart

Rédactrice en chef:

Muriel Dejemeppe

Secrétariat \& logistique:

Virginie Leblanc

Graphiste:

Dominique Snyers
Comité de rédaction:

Paul Belleflamme

Vincent Bodart

Muriel Dejemeppe

Gilles Grandjean

Jean Hindriks

William Parienté

Sandy Tubeuf

Frédéric Vrins

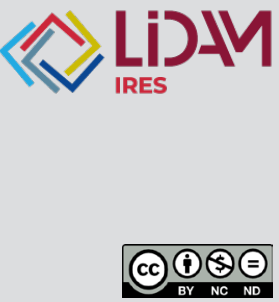

